\title{
A snapshot review of culturally competent compassion as addressed in selected mental health textbooks for undergraduate nursing students
}

\author{
Irena Papadopoulos ${ }^{*}$ and Alfonso Pezzella
}

\begin{abstract}
Background: The recent scandals involving poor healthcare put nurses under the spotlight in an attempt to understand how compassionate they are towards their patients.

The aim of this article is to investigate how compassion is embedded in the textbooks of the undergraduate mental health nursing degree.

Methods: A snapshot review of key textbooks used, was conducted through the distribution of a list of textbooks and search terms to a panel of mental health teachers in four United Kingdom (UK) universities. They were asked to comment on the list's completeness, and the terms' suitability, comprehensiveness and sensitivity regarding culturally competent and compassionate care.

Relevant data were extracted independently by each author followed by meetings to compare and discuss their findings and engage in deeper levels of analysis.

Results: The review found that despite the fact that few textbooks touched on a number of the search terms none of them directly addressed the issue of compassion or culturally competent compassion. This means that mental health undergraduate nurses may not be adequately prepared to provide culturally competent compassion.

Conclusions: Culturally competent compassion is not something we are born with. Imaginative teaching methods, good textbooks, good role models and opportunities to practice what one learns under supervision is required to nurture compassion in order to re-establish itself as the essence of nursing. Key textbooks need to be revised to reflect the virtue of culturally competent compassion.
\end{abstract}

Keywords: Compassion, Culturally competent compassion, Mental health nursing, Mental health nursing textbooks

\section{Accessible summary}

What is known on the subject

- Little is known whether resources such as key textbooks provide adequate information to help nursing students to learn about compassion and in particular culturally competent compassion.

- Compassion is currently being debated in nursing following numerous reports about poor nursing care.

* Correspondence: r.papadopoulos@gmail.com

Research Centre for Transcultural Studies in Health, Middlesex University, Hendon Campus, The Burroughs, London NW4 4BT, UK
- The Department of Health has declared in their new vision and strategy for nurses, midwives and care givers that compassion should be at the centre of care.

\section{What this paper adds to existing knowledge}

- The review found that mental health textbooks used in many undergraduate curricula include some coverage of topics related to compassion but none of them directly address the issue of compassion or culturally competent compassion.

- Textbooks need to reflect the virtue of culturally competent compassion and contribute to its learning. 


\section{What are the implications for practice}

- The findings indicate that mental health nursing education needs textbooks which explicitly and fully deal with culturally competent compassion.

- In order to achieve adequate coverage of culturally competent compassion in key mental health nursing textbooks, nursing research policy and funding needs to be reviewed.

\section{Background}

This article is an attempt to review whether and how UK mental health nursing students are being prepared to learn not just about compassion but about 'culturally competent compassion' [1] by reviewing the key textbooks used in the undergraduate curriculum.

The recent reports and growing evidence about poor standards of nursing care, particularly with vulnerable people, have suggested that compassion in nursing and generally in the provision of National Health Services (NHS) is often an aspiration and not a true reflection of practice [2-5]. In response to the alarming findings catalogued in these and other inquiries, as well as articles in the mass and online media, three key reports have been published: the 'Willis Commission on nursing education' [6], the 'Compassion in Practice. Nursing, midwifery and care staff. Our vision and strategy' ([7],) and the 'Raising standards, putting people first. Strategy for 2013-16' [8]. Compassion is the common theme in all of them.

These reports coexist with the consensus in the recently published literature that healthcare is becoming more complex and the demands for it are ever increasing. Questions have been raised as to whether the current educational preparation of nurses adequately equips them for their work. The published literature regularly refers to these factors as having an impact on the nurses' ability to provide high quality compassionate care to all patients. There is however one factor which is missing from the recent debate. This is the fact that society in the UK, (and in many other countries) is multicultural, and consequently the nursing and healthcare teams are increasingly becoming multicultural too. The lack of attention to this crucial fact leads us to suggest that those responsible for education as well as managing healthcare teams assume that compassion is void of cultural meaning and expressions. The Willis report on nursing education [6] is the exception as it provides a small quotation from the submission to its commission by Professor Irena Papadopoulos in which she states:

"Britain is a multicultural country and nurses must be able to provide clinically competent but also culturally competent and compassionate care. We should invest in multicultural teams working for students, clinical teams and nurse educators" (p.35).

Papadopoulos [1] has defined culturally competent compassion as the human quality of understanding the suffering of others and wanting to do something about it using culturally appropriate and acceptable nursing/healthcare interventions which take into consideration both the patients' and the carers' cultural backgrounds as well as the context in which care is given. The specific focus for this review is the key textbooks used in mental health nursing. We acknowledge that other learning materials such as published articles, information available on the internet, as well as materials and activities designed by teachers which may focus on practice learning are used.

\section{Project aim and research question}

The project aims to explore how culturally competent compassion is taught and embedded in the undergraduate mental health nursing curriculum. We are investigating, "How are mental health nursing students being prepared to gain theoretical knowledge and understanding regarding culturally competent compassion during their undergraduate programme with specific focus the key texbook used?"

\section{Method/design \\ Snapshot review}

The project adopted a snapshot review approach of the main key textbooks used in the undergraduate mental health nursing degree.

A snapshot study is carried out over a very short period of time, and invariably provides an overview of the state of something, in a particular time.

(Macmillan dictionary. http://www.macmillandictionary. com/dictionary/british/snapshot).

A list of textbooks was constructed based on the reading lists in the mental health undergraduate nursing curriculum at a London based university. In addition to this, a list of search terms that would answer the research question was produced.

A panel of experts from three other UK-based universities were sent the list of textbooks and search terms and were invited to respond to the following questions:

"In your view, is this an adequate list of textbooks for the undergraduate mental health nursing degree? YES/NO"

"In your opinion, are there any other key textbooks that are missing from this list but appear in the list of the mental health nursing curriculum in your university? 
In your view, are these textbooks used widely in mental health nursing curriculum of UK universities?

(Please supply full references)".

Their comments were also sought in relation to the identified search key terms. The panel were asked to comment on: 1) the suitability, 2) the sensitivity and 3) the comprehensiveness of the textbooks being reviewed.

For the full list of search key terms see Table 1 and for the full list of textbooks see Table 2.

Once, the final list of key textbooks used nationally was agreed, as well as that of the key terms, following the feedback from the expert panel, the authors searched the contents and subject index pages of each textbook on the list, in order to identify texts which related to 'compassion' and "culturally competent compassion".

\section{Capturing the data}

An extraction table, with the textbook references, the key terms and a column for comments was created. One of the authors went through the contents and index pages of the sourced books noting the key terms found and the related page number/s. In order to assure the quality and reliability of the extracted data the other author also repeated the exercise adding any new data found.

Following this stage of data extraction, both authors met to discuss the findings and to engage in a deeper level of analysis which consisted in locating each key term found in the main body of the book, and making comments regarding the extend of use of the key term, for example, whether it was a definition, a paragraph or whether a whole chapter was devoted to it.

Table 1 List of key search terms

\begin{tabular}{ll}
\hline Cultural competence related terms & Compassion related terms \\
\hline Cultural competence & Compassionate caring \\
Culturally Competent compassion & Compassion \\
Culture & $\begin{array}{l}\text { Compassion and mental } \\
\text { health nursing }\end{array}$ \\
Diversity & Dignity and compassion \\
Ethnicity & Empathy \\
Marginalised & Learning compassion \\
Multicultural & Measuring compassion \\
Transcultural nursing & Practising compassion \\
& Suffering \\
& Sympathy \\
& Theories of compassion \\
& Values and compassion \\
\hline
\end{tabular}

Comments about the total number of pages for each book, the total number of chapters in each book and the depth of coverage of each key term were also noted (Table 2).

A certain level of flexibility needed to be applied to this process due to the fact that although quite a few of the key terms were not directly quoted or used, it became evident that content relating to key terms was being addressed. For example, in one of the books, mental health nursing values were discussed and although their relationship to compassion was obvious, the term compassion was never used. In such cases the authors opted to credit the content to the nearest key term.

\section{Results and discussion}

The findings of this review, summarised in Table 2, revealed that despite the fact that some of the textbooks had content on ethnicity, diversity, empathy, sympathy, dignity, culture, cultural competence, transcultural nursing, multiculturalism, marginalisation, suffering, values and compassion, ranging from a brief definition to one or two short paragraphs, none of them directly addressed the meaning of compassion and culturally competent compassion, its relevance to nursing, its impact on the patients, the strategies of learning and applying culturally competent compassion, and so on.

The terms "culturally competent compassion", "theories of compassion" "practising compassion", "measuring compassion", "learning compassion" and "compassion and mental health nursing" were not identified in any of the content and index lists of the key textbooks we reviewed.

Despite the mass media and the emphasis of the new health guidelines, on the need to be compassionate and culturally competent towards patients, the findings of this review demonstrate that culturally competent compassion is not directly addressed in the key textbooks used in the undergraduate mental health nursing degree. This means that mental health undergraduate nurses may not be adequately prepared to provide culturally competent compassion. We would, however, like to acknowledge that although textbooks provide a strong indication as to what nurse educators value and use, our experience tells us that in addition to textbooks, published articles are also used which may fill the compassion gap. Teachers frequently use practice based scenarios and other methods to promote compassion and cultural competence. However, we believe that there is an urgent need to develop learning tools to be used by students, teachers and practitioners for the development of knowledge, attitudes and skills appropriate to culturally competent compassion in a more systematic way.

Papadopoulos [9] has developed a conceptual model for culturally competent compassion based on the Papadopoulos 
Table 2 List of textbooks and analysis

Textbooks

(editors) (2009) Foundation studies for caring.

Barker, P. (2009). Psychiatric and Menta Health Nursing: The Craft of Caring. (2nd Ed). UK: Hodder Arnold.

Norman, I and Ryrie (2013). The Art and Science of Mental Health Nursing. Maidenhead: McGraw Hill
Basingstoke Palgrave Macmillan

Key terms and citation number of pages

Diversity (541)

Culture $(94-104,674)$

Ethnicity $(98,184,601)$

Marginalised (671)

Mindfulness (384, 385-386, 389, 610)

Sympathy (323)

Culture $(72,201-202,610,642)$

Ethnicity (636-639, 641-642, 722-723)

Multicultural (359-360, 508, 511-513,

640-641)

Empathy $(151,153,281-282,321-329$

323,331 )

Cultural competence (510-511, 635,

638-641)

Values $(13,609-612,732)$

Compassion $(665,270-271,307-398)$

Cross cultural (389-390)

Ethnicity $(29,492)$

Empathy $(272,308)$

Compassionate caring (171-182)

Dignity (160-162)

\section{Compassion (43)}

Bach, S. and Grant, A. (2009). Communication and Interpersonal Skills for Nurses. Poole: Learning Matters.
Transcultural nursing (125)

Diversity $(38,141-142,145-151,156-163$

Culture $(129,141,168$

Ethnicity $(129,168,147,137,151-152)$

Multicultural $(146,151-156,160,125$, 134-136)

Empathy $(37-38,53-56,59,168)$

Cultural competence $(125$, Page 152

Page 154-155)

Dignity (10)

Marginalised (162-163)

Summary

The book has 740 pages and it is divided in 4 parts. Relevant chapters are "Ethical Legal and Professional

Issue" (chapter 4) "Communication" (chapter 5) and "Culture" (chapter 6). The chapter on culture also mentions about ethnicity and culture. Chapter 26 (Mental Health) has brief mention about diversity, while chapter 33 (HIV and Infectious disease management) mentions marginalisation.

The book has 757 pages with a comprehensive list of contents and a very comprehensive index of 16 pages. It is divided in 10 sections, and a total of 84 chapters. It contains a whole chapter on "Developing Empathy" (chapter 37). A whole chapter on "Mindfulness" (chapter 45). A whole chapter on "Services for Asylum Seekers and Refugees" (chapter 59). A whole section on "Legal Ethical and Moral Issue" (section 8).

The book has 389 pages and it is divided in 6 parts and 42 chapters.

Chapter 12 deals with "Therapeutic relationships" and chapter 13 with "Creating therapeutic environments" Compassion, ethnicity and empathy are briefly mentioned in chapter 2 (mental disorder). Cultural sensitivity is mentioned in chapter 9 (the service context and organization of mental health care) and chapter 32 (older people with functional mental health problems). Immigration and Racism in chapter 8 (the social policy context of mental health care). Dignity is dealt in chapter 11 (the ethic of mental health nursing). Empathy is also mentioned in chapter 17 (Assessing and managing the risk of self-harm and suicide). Ethnicity is mentioned in chapter 32. Cross-cultural working is mentioned in chapter 25 (working with families and carers). Compassion (diagram dealing with quality of nursing in care) is mentioned in chapter 42 (Oder People are the Future).

The book is 184 pages and contains 8 chapters. The list of contents only contains the titles of the chapters and it is not broken down in more extensive list of concepts within the chapters unlike the other books examined.

Chapter 2 (Key Concepts) deals extensively with suffering, empathy, diversity and healthy relating through a number of reflective activities. It also provides references for further reading in compassion and the website, compassionate mind.

Chapter 4 (Safe and Effective Practise) deals with nursing-patient relationships. Chapter 5 (Understanding Potential Barriers) deals with the emotional context of communicating. Chapter 7 (The Environmental Context) deals with multidisciplinary, team practise and Interprofessional working. Chapter 8, (Population and Diversity Context) deals with migration, diversity, culture, communicating with cultural diversity, multicultural Britain cultural competence, transcultural nursing. The book has a useful glossary, which contains all the terms mentioned above. 
Table 2 List of textbooks and analysis (Continued)

Burnard, P. and Gill, P. (2009). Culture, Communication and Nursing: A Multicultural Guide. Harlow: Pearson Education Ltd.

Schultz, J.M. and Videbeck, S.L. (2008) Lippincott's Manual of Psychiatric Nursing Care Plans. (8th Ed) Philadelphia: Lippincott, Williams \& Wilkins.

Nash, M (2010) Physical health and well-being in mental health nursing: clinical skills for practice Maidenhead: Open University Press

Slade, M. (2009). Personal Recovery and Mental IIIness: A guide for mental health professionals. Cambridge University Press

Clarke. V. and Walsh, A. (2009) Fundamentals of Mental Health Nursing. Oxford University Press

Benthall, R.P., 2003, Madness explained London: Penguin

Watters, E., 2010, Crazy like us London: Constable \& Robinson

\section{Suffering $(32-33,170)$}

Transcultural nursing $(27,158,159$, 160, 166)

Ethnicity (14)

Empathy (52, 153-154,)

Culture (13-15)

Culture $(62,139)$

Marginalised (7)

None

Transcultural nursing (170-171)

Diversity (280)

Multicultural (167, 187-189)

Empathy $(28-29,105)$

Values $(4,7-8,277)$

Culture (130-131, 139, 519-520, 180 $356,357,214-214,236-237,300)$

Multicultural (130-133)

Empathy (28-29)

Marginalised (413-414)

Empathy (161)
The book has 181 pages; it is divided in 8 chapters. The whole book discusses culture, communication and nursing. There are no references to compassion nor to other compassion related terms we used except empathy.

The book has 439 pages and it is divided in three parts. Part 1 - using the manual; Part 2 - key consideration in mental health nursing and Part 3 contains 13 sections, with each sections containing a number of care plans.

Under sub-section titled culture, notions of cultural background, cultural awareness, cultural differences in needs, cultural orientation, dominant and minority cultural, ethno-centricity, cultural differences, obstacles to cross-cultural care, delivering cultural sensitive care are discussed in 4 columns equivalent to two pages. There is no mention of compassion.

The book has 222 pages and it contains 10 chapters. The book contains two small paragraphs on inequalities in health (chapter 1). Cultural preferences form one of the components on a diagram of physical assessment (chapter 4). Three small paragraphs deal with cultural and religious factors (chapter 7).

The book has 275 pages and it is divided in 4 sections and 26 Chapters. Although this book has a chapter on social inclusion and a section on diversity models and discriminations, neither of these address the cultural perspective. It also has a page on religion. The book contains a list of values expected from mental health workers but nothing about compassion included.

The book has 289 pages and it contains 10 chapters. No relevant concepts are included in the contents list. Chapter 1 (Becoming a Student Mental Health Nurse) deals with values, believes and the ethical code of nursing. Values and attitudes are also addressed in chapter 10 (On Becoming a Qualified Mental Health Nurse). Empathy is mentioned in chapter 2 (Fundamental Skills of Mental Health Nursing) and chapter 5 (The Nursing Care of an Adult in Crisis with Mental Health Problems). Multiculturalism is dealt with it in a clinical scenario in chapter 7 (The Nursing Care of Older People with Mental Health Problems) and chapter 8 (Mental Health Nursing in the Community).

Diversity appears as a definition in the glossary. Transcultural nursing is mentioned in a clinical scenario in Chapter 7.

The book has 640 and it is divided in 4 parts and has 19 chapters. The index is highly populated with author's names and fewer concepts and terms. It may therefore contain cultural and compassion elements in the pages describing the works of the various theorists (unlikely). Chapter 6, titled "Them and Us", contains a section entitled "Cross cultural differences and symptoms". It also contain a section on "culture and psychiatric illness". Chapter 9 (Madness and Emotion) covers briefly culture and emotions. Chapter 10 (depression and pathology of self) covers briefly culture and depression. Chapter 12 (abnormal attitudes) covers briefly culture and delusion. Chapter 14 (the illusion of reality) covers briefly culture and hallucinations.

Chapter 2 (After Kraepelin) covers briefly empathy.

Chapter 16 (Things are much more complex than they seem) covers briefly social isolation.

The book has 306 pages and it contains 4 chapters plus an introduction and conclusion. (Unlike all the authors listed in this table, the author is an American journalist. The book concentrates mainly on anorexia, PTSD, schizophrenia and depression and provides information about the cultural expressions of these in numerous countries in the world. It does not address compassion.

Contains information about culture-bound syndrome of depression; diversity of cultural expression of depression, culture sadness, indiums, Islam, cultural-language deficiencies, beliefs. 
Table $\mathbf{2}$ List of textbooks and analysis (Continued)

Gamble, C \& Brennan G (2006) Working

with Serious Mental Illness 2nd edition Elsevier

\section{Ethnicity (360)}

Culture $(360-369)$

Empathy (74-75)

Compassion and mental health (73-85)

Transcultural nursing (29)

Empathy (60-61)

Benthall, R. P (2009) Doctoring the Mind:

Why Psychiatric Treatments Fail Penguin
The book has 427 pages and it is divided in four sections with 22 chapters. Chapter 3 (Social Inclusion).

Chapter 6 (Building Relationship, Lesson to be Learnt). Chapter 19 titled Cultural Issues. Chapter 20 "Ethical Considerations" (mainly about confidentiality and consent).
The book has 364 pages and it is divided in three parts. Two line reference to cross-cultural psychiatry.

Briefly describes Karl Rogers empathetic understanding. The book focuses on mental illness and psychiatric intervention. 


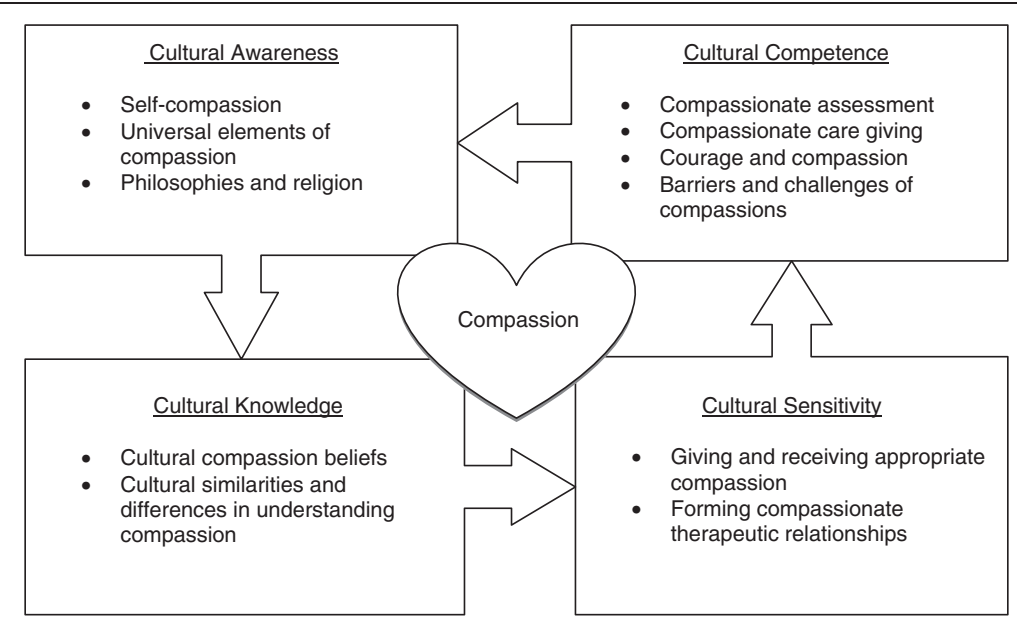

Fig. 1 The Papadopoulos model for developing culturally competent and compassionate healthcare professionals

[10, 11]; Papadopoulos et al. [11] model of cultural competence. In the absence of any other models, we present this here as a suggestion (Fig. 1).

In summary, according to this model culturally competent compassion begins with an awareness of our self compassion. This according to Neff [12] involves treating ourselves with kindness, caring, nurturance, and concern, rather than being harshly judgmental or indifferent to our suffering and vulnerabilities. However universal these emotions and behaviours may sound, they are not all equally valued by cultures and religions. It is therefore important that students are encouraged to learn and discuss the similarities and differences which exist among cultures in the way compassion is understood and expressed. This will help them establish sensitive and culturally appropriate compassionate relationships with the patients in their care. Such relationships are the vehicles to culturally competent and compassionate assessments of the needs and suffering which is associated with illness. The ultimate expression of culturally competent compassion is the demonstration of courage at times when barriers to compassion -for whatever reason - are created which must be challenged and eliminated.

\section{Conclusions}

The kind of compassion alluded to above is not something we are born with, neither is a 'walk in the park' type of learning, as it requires time, imaginative teaching methods, good resources such as textbooks, good role models and opportunities to practice what one learns under supervision. With time and practice, the desired culturally competent compassion habits and associated wisdom will develop. Nursing needs to nurture compassion, to help it flourish and re-establish itself as the essence of the profession. Textbooks need to reflect the virtue of culturally competent compassion and contribute to its learning.

\section{Competing interests}

The authors declare that they have no competing interests.

\section{Authors' contributions}

IP conceived and directed the project which this article is based on. AP sourced all the materials and communicated with the panel of experts. IP and AP conducted the analysis and drafted the article. Both authors read and approved the final manuscript.

Received: 22 December 2014 Accepted: 11 May 2015

Published online: 22 May 2015

\section{References}

1. Papadopoulos I. Courage, Compassion and Cultural Competence. The 13th Anna Reynvaan Lecture. 19th May 2011, De Stadsschouwburg - Amsterdam City Theatre. Netherlands. 2011.

2. Mullan K. Patients not numbers. London: People not Statistics. The Patients' Association; 2009.

3. The Mid Staffordshire NHS Foundation Trust Inquiry. The Independent inquiry into care provided by Mid Staffordshire NHS Foundation Trust January 2005-March 2009. London: The Stationary Office; 2010.

4. Parliamentary and Health Services Ombudsman. Care and compassion? Report of the Health Service Ombudsman on ten investigations into NHS care of older people. London: The Stationery Office; 2011.

5. The Mid Staffordshire NHS Foundation Public Inquiry. Report of the Mid Staffordshire NHS Foundation Trust Public Inquiry Stationary Office, London. 2013.

6. Royal College of Nursing. Quality with compassion: the future of nursing education. Report of the Willis Commission on Nursing Education. London: RCN; 2012.

7. Department of Health. Developing the culture of compassionate care: creating a new vision and strategy for nurses, midwives and care givers. 2012. Retrieved from http://consultations.dh.gov.uk/nhscb/47269592 on 24 February 2014.

8. Care Quality Commission. Raising standards, putting people first. Strategy for 2013-16. CQC, Newcastle Upon Tyne. 2013.

9. Papadopoulos I. Papadopoulos model for culturally competent compassion. 2014. https://www.youtube.com/watch?v=zjKzO94TevA.

10. Papadopoulos I, editor. Transcultural Health and Social Care: Development of Culturally Competent Practitioners. Edinburgh: Churchill Livingstone Elsevier; 2006.

11. Papadopoulos I, Tilki M, Taylor G. Transcultural care. A guide for health care professionals. Dinton, Wilts: Quay Publications; 1998.

12. Neff KD. Self-compassion, self-esteem, and well-being. Soc Pers Psychol Compass. 2011;5:1-12. 